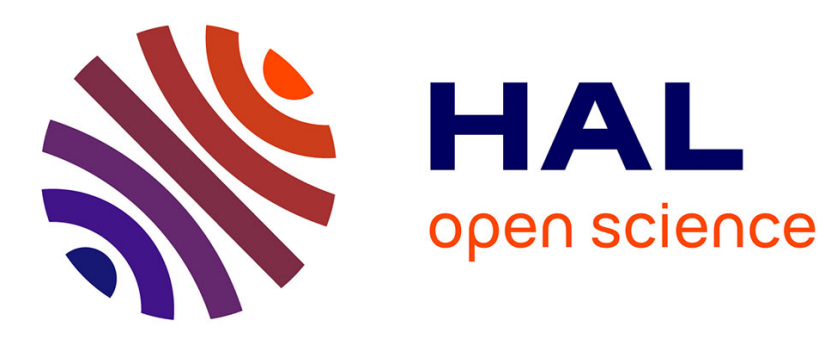

\title{
Nonlinear retinal response modeling for future neuromorphic instrumentation
}

Jason K Eshraghian, Seungbum Baek, Timothée Levi, Takashi Kohno, Said Al-Sarawi, Philip H W Leong, Kyoungrok Cho, Derek Abbott, Omid Kavehei

\section{- To cite this version:}

Jason K Eshraghian, Seungbum Baek, Timothée Levi, Takashi Kohno, Said Al-Sarawi, et al.. Nonlinear retinal response modeling for future neuromorphic instrumentation. IEEE Instrumentation and Measurement Magazine, 2020, 23 (1), pp.21-29. 10.1109/MIM.2020.8979519 . hal-02484026

\section{HAL Id: hal-02484026 \\ https://hal.science/hal-02484026}

Submitted on 25 Feb 2020

HAL is a multi-disciplinary open access archive for the deposit and dissemination of scientific research documents, whether they are published or not. The documents may come from teaching and research institutions in France or abroad, or from public or private research centers.
L'archive ouverte pluridisciplinaire HAL, est destinée au dépôt et à la diffusion de documents scientifiques de niveau recherche, publiés ou non, émanant des établissements d'enseignement et de recherche français ou étrangers, des laboratoires publics ou privés. 


\section{Nonlinear Retinal Response Modeling for Future Neuromorphic Instrumentation}

Jason K. Eshraghian, Seungbum Baek, Timothée Levi, Takashi Kohno, Said Al-Sarawi, Philip Leong, Kyoungrok Cho, Derek Abbott and Omid Kavehei

The development of a bio-inspired image sensor that can match the functionality of the vertebrate retina in terms of image resolution, power dissipation and dynamic resolution demands an extremely challenging standard in terms of efficiency and performance $[1,2,3]$. At present, functional biological image processing possesses extremely high temporal resolution by asynchronously optimizing the sampling rate of a scene but suffer from low spatial resolution, noise, and an inability to process low frequency content [4]. Regardless, the use of dynamic vision sensors that emulate biological retina behaviors are important in performing classification tasks using high-speed motion detection at low-power requirements $[5,6,7]$. The difficulty in pushing image sensor performance to meet the specifications of the retina lies in the biological complexities of the underlying neural networks. Therefore, a prerequisite for hardware mapping of biological vision systems is understanding the bioprocessing between various retina cells. Here, we present a biologically plausible cellular network retina simulator where light stimuli are accepted at the front-end array of photoreceptor cells, and pass through a system of nonlinear integral equations derived from experimental voltage- and current-clamp data used to calculate the response of each sequential retina cell. It was shown in [8] that integrating the system improves performance when compared with conventional numerical solvers. We expect that by placing our simulator in the hands of interdisciplinary researchers, especially computational neuroscientists, mathematical scientists and machine learning practitioners, it will foster the development of more efficient representations of visual inputs in deep learning applications.

\section{Retinomorphic Processing}

The retina can be approximated into five levels of cellular layers through which incident light passes: the rod and cone photoreceptors (outer boundary of the retina), horizontal cells, bipolar cells, amacrine cells and ganglion cells (inner boundary). When light enters the eye, it encounters two types of photoreceptors: the rod cells (highly sensitive in photopic conditions and that enable color vision) which convert light into nerve signals [9] and the cone cells (sensitive in scotopic conitions and assist with vision in dimly lit scenes). In retina modeling the electromagnetic frequency of a photon is often ignored due to the significant complexity 
associated with measurement of spectral sensitivity in a photoreceptor mosaic, in conjunction with simulating a large-scale cellular network. In the RGB color space, this would triple the throughput of a system. However, with the development of techniques that significantly reduce the computational resources needed for spike-train generation such as pre-integration of compartmental retina models, we can demonstrate a real-time retinal mode that reduces simulation runtimes by up to $50 \%$ over equivalent state-of-the-art methods without compromising signal accuracy in using larger time steps. Notably, this model not only produces ganglionic cell spike-trains that are dependent upon the intensity and wavelength of incoming light, but also the intermediary action potentials and graded potentials produced by the electrical and chemical intra- and inter-cellular interactions.

Biologically plausible models of image capture and processing seek to bridge the physical gap between sensing and computation. In doing so, advantages in comparison to traditional cameraprocessor approaches manifest in the form of heightened parallelism, small chip size and low power dissipation, and high dynamic range [10], [11]. To achieve this standard of neuromorphic hardware implementation, there are several barriers to overcome. These include the lack of access to experimental data from the vertebrate retina, the difficulty of processing large-scale compartmental models without introducing simplified assumptions in real-time, and the limited interconnectivity in silicon across a 2D planar chip.

In this work, we seek to address the issue of real-time processing without compromising accuracy. We provide open-access to a retinal simulation platform for researchers at the end of this paper, to facilitate a wholly integrated real-time model of a measurable retinal network with adaptive parameters, including a variation of cell membrane capacitance, maximum conductance, reversal potential, ion channel selection, and electrical/chemical synaptic parameterization. We seek to open up the frontiers for accessible and reproducible benchmarks in neuromorphic instrumentation for vision processing, in both individual and populations of neuronal measurements to provide a more in-depth insight into the role of the various physical, chemical and electrical properties of the retinal cellular network. We will first demonstrate the high-level architecture of our retinal measurement platform as depicted in Fig. 1 before looking at the constituent low-level compartmental blocks, and the resultant data procured from the retinal network. 


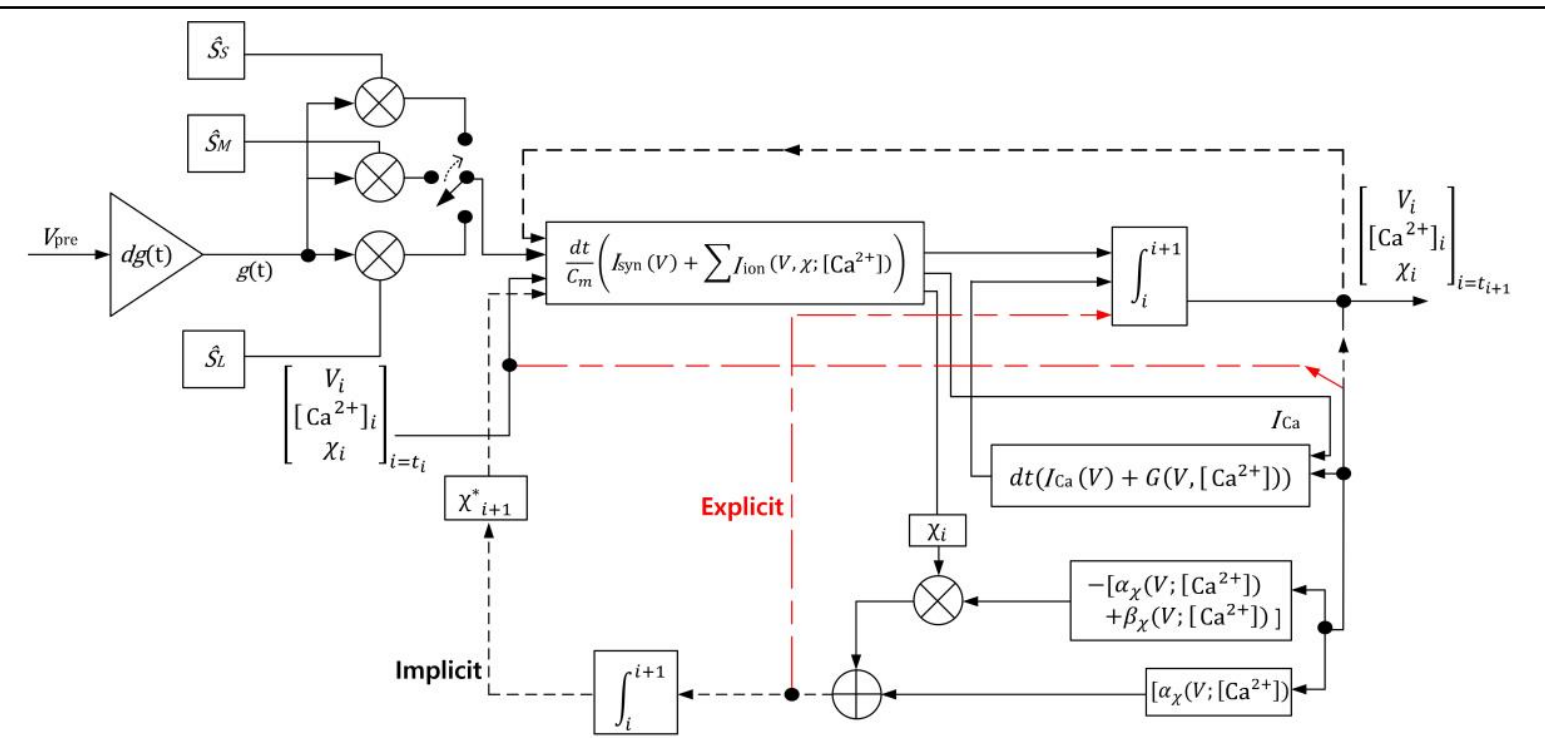

Fig. 1 Higher-level architecture of photocurrent signal processing and computation.

\section{Signal Processing and Computation Architecture}

With higher precision and greater parameterization comes slower processing. Computer vision algorithms are often implemented without regard to the computer architecture or hardware they are designed to run on, and so similar structures and principles should ideally exist between algorithm and hardware. With the construction of a large system of integral equations it becomes necessary to accelerate computational efficiency, where from a mathematical perspective, a system of ordinary differential equations (ODEs) and integral equations describe identical phenomena. However, from an algorithmic-hardware co-design analysis, three salient benefits arise:

- Ability to solve the large system of nonlinear integral equations in real-time using small time-steps of sub-nanosecond order.

- Reduced reliance on off-chip memory, as hardware implementation of derivatives require memory and a delay line, whereas our integral formulation only requires summations for correct implementation.

- Biophysically accurate representation of the retina at a cellular level.

The formulation of a conductance-based compartmental retinal model initiates at the first input layer of photoreceptor cells, passes through three hidden layers, and triggers the output ganglion cells which respond with a spike-train sent to the visual cortex for further processing and interpretation. The system of ODEs can be generically characterized by: 


$$
\begin{gathered}
C_{m} \frac{d V}{d t}=\sum I_{\mathrm{ion}}\left(V, \chi ;\left[\mathrm{Ca}^{2+}\right]\right)+I_{\mathrm{syn}}(V), \\
\frac{d\left[\mathrm{Ca}^{2+}\right]}{d t}=I_{\mathrm{Ca}}(V)+G\left(V ;\left[\mathrm{Ca}^{2+}\right]\right), \\
\frac{d \chi}{d t}=(1-\chi) \alpha_{\chi}(V)-\beta_{\chi}(V) \chi
\end{gathered}
$$

where $C_{m}$ is the membrane capacitance, $V$ is the membrane potential, $I_{\text {syn }}(V)=g(\mathrm{t})\left(E_{\text {syn }}-\right.$ $V$ ) is the synaptic current input, $E_{\mathrm{syn}}$ is the reversal potential, and $I_{\text {ion }}$ is the sum of all ionic voltage and calcium-dependent ionic currents. Here, $\left[\mathrm{Ca}^{2+}\right]$ is the intracellular concentration of free calcium, $I_{C a}(V)$ denotes all voltage-activated calcium currents, while $G$ represents all other voltage and calcium-dependent cellular processes. The gating variable is denoted $\chi$, and forward-backwards propagation rates are represented by $\alpha_{\chi}$ and $\beta_{\chi}$, respectively. While the complete and detailed system of equations, initial conditions, biasing and physical descriptions are subsumed within the high-level description, their details can be found in an online appendix with a link provided in the references [12].

The signal processing architecture of the integrated form of equations (1) - (3) is shown in Fig. 1. The architecture provides a visual understanding of how the system of nonlinear integral equations behaves within a nonlinear time-invariant system (that is, the light input is the timevarying component measured in the number of rhodopsin, $\mathrm{Rh}^{*}$ in Fig. 2, and photoisomerization molecules activated per second).

The computational mapping of a photocurrent signal from the input photoreceptor layer through to the ganglion cell output layer requires an input conductance $g(t)$ that is weighted by the spectral sensitivity of the cone cell it comes into contact with. The resulting signal is passed to the primary voltage/ionic current evolution block where it is used to derive the synaptic current. The summation of currents and gating variables is fed to the total calcium current in a secondary block that describes the evolution of calcium concentration. The signals are combined and integrated to generate new values for membrane potential and calcium at the next time step $t_{i+1}$. This formulates the high-level architecture for the retinal network. A sequential demonstration of the first two layers of photoreceptor (rod and cone) and bipolar cells is provided in Fig. 2. 


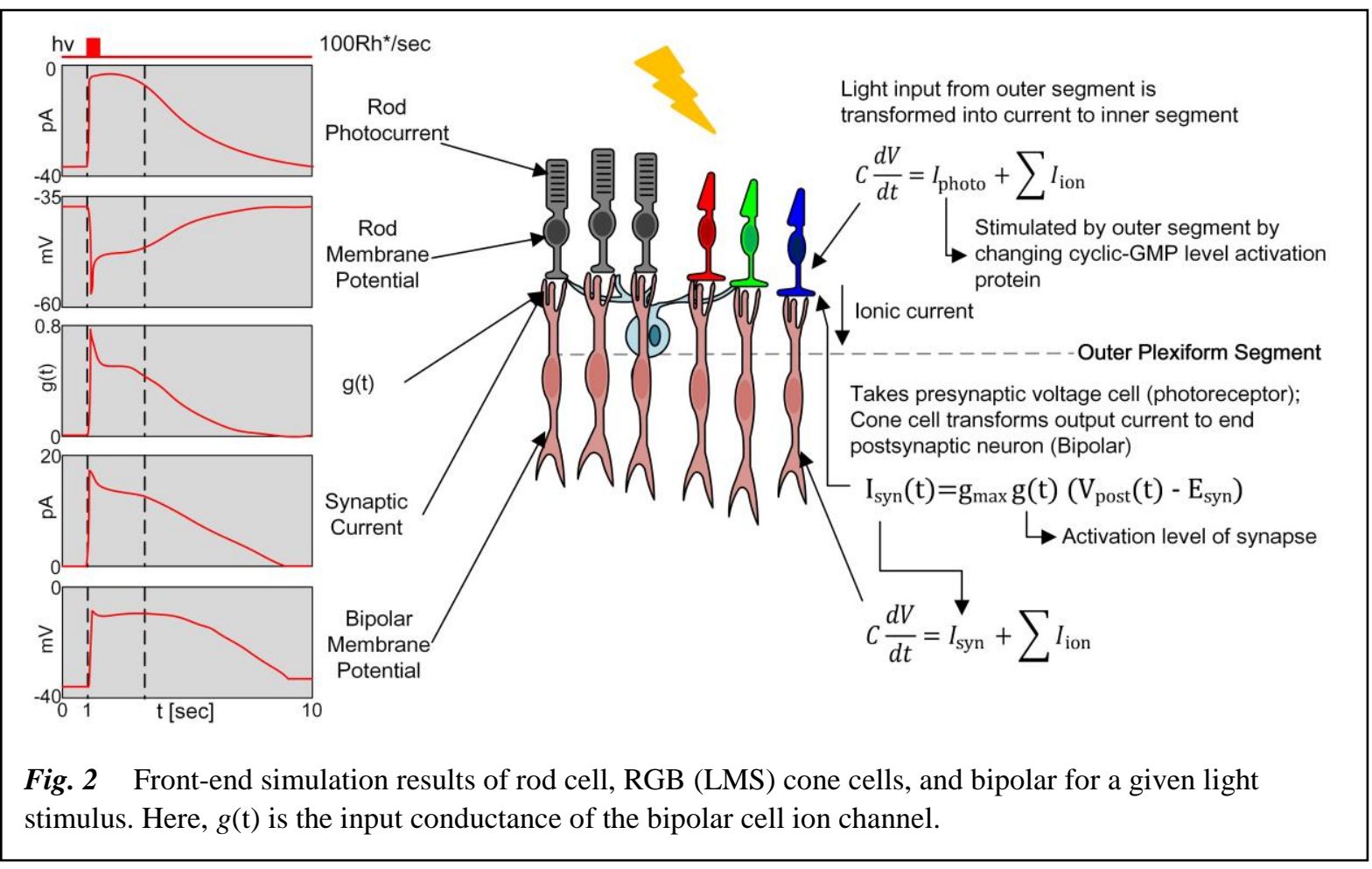

\section{Retinal Model}

\section{Spectral Sensitivity}

Light incident on the retina is a mixture of different wavelengths, and at least three different subclasses of cone photoreceptor cells have been experimentally verified to be wavelengthdependent. Each of these subclasses can be classified based on the wavelength sensitivity of the photopigment in its outer segment: long-wavelength (L), middle-wavelength (M) and shortwavelength (S) peak sensitive cone cells.

The photoreceptor mosaic of varying spectral sensitivities is implemented in the proposed model as input dependent weighting coefficients, where normalized sensitivity is represented by weights $\hat{S}_{\mathrm{S}}, \hat{S}_{\mathrm{M}}$ and $\hat{S}_{\mathrm{L}}$ that are approximate models of cone cell responses across the spectrum of visible wavelength, based on corneal measurements presented in [13]. These models were fit using least squares regression with the $5^{\text {th }}$ order polynomial, which quantitatively match the precision of the rest of the model:

$$
\hat{S}_{S}=\left\{\begin{array}{cc}
-1.3 \times 10^{-9}+3.1 \times 10^{-6} \lambda^{4}-2.9 \times 10^{-3} \lambda^{3} \\
+1.3 \lambda^{2}-310 \lambda+2.9 \times 10^{4}, & 400 \leq \lambda \leq 530 \\
0, & \text { otherwise }
\end{array}\right.
$$




$$
\begin{gathered}
\hat{S}_{M}=\left\{\begin{array}{lr}
-0.099 z^{5}+0.24 z^{5}-0.4 z^{3}+0.94 z^{2} \\
-0.37 z+0.87, & 400 \leq \lambda \leq 530 \\
0, & \text { otherwise }
\end{array}\right. \\
\hat{S}_{L}=\left\{\begin{array}{lr}
0.12 y^{5}+0.21 y^{4}-0.55 y^{3}-0.88 y^{2} \\
+0.59 y+0.9, & 450 \leq \lambda \leq 620 \\
0, & \text { otherwise }
\end{array}\right.
\end{gathered}
$$

where $\lambda$ is wavelength in nanometres, $z=\frac{\lambda-5.4 \times 10^{2}}{76}$ and $y=\frac{\lambda-5.5 \times 10^{2}}{85}$. Another reason for using polynomial forms, more generally, is the simplicity of using artificial neural networks to encode polynomial functions, which becomes essential for deep learning applications and predictive retinal networks.

The cone cell variations in spectral sensitivity demonstrate that the biological retina does not respond equally to all types of light, and does not have a uniform sensitivity across the visible spectrum. In addition to this, there is further variation across the photoreceptor mosaic (our field of view) to light intensity where the fovea is considered to be the centre. Therefore, the Westheimer linespread function can be used to capture the characteristics of polychromatic light more accurately:

$$
\hat{\mathrm{S}}=0.47 e^{-3.3 i^{2}}+0.53 e^{-0.93|i|},
$$

which now introduces a dependence on not only photon wavelength but also on the position upon the photoreceptor array where the light impinges, where $i$ is the position on the retina (on a 2-dimensional axis) specified in terms of minutes of visual angle from the fovea. We can calculate the different weights based on input wavelength $\lambda$ and position on which light falls on the retina $i$ for a short-wavelength peak sensitive cone cell by taking the root of (4), and multiplying it by (7), which has been graphically illustrated in Fig. 3.

This is a near perfect fit of the benchmark data of spectral sensitivities across the photoreceptor mosaic in [14], and also demonstrates that peak sensitivity occurs at $i=0$ for a wavelength of $\lambda=440 \mathrm{~nm}$. To qualitatively summarize the function of (4) - (7), the input light is weighted using our spectral sensitivity model based on the following three features:

- whether light targets a L, M or S cone cell;

- the wavelength of the incoming light, and

- the visual angle of incoming light concerning the targeted cell. 


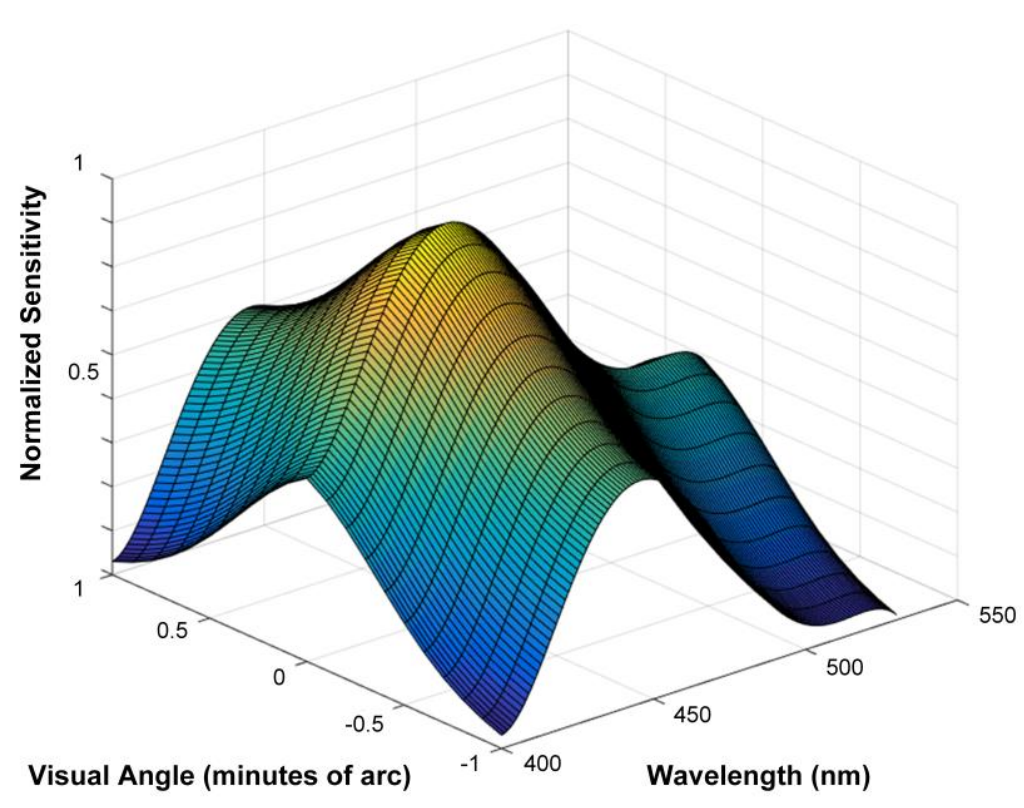

Fig. 3 Simulation of normalized spectral sensitivity as a function of light wavelength and the visual angle away from the targeted $\mathrm{S}$-cone cell as per the proposed model.

\section{A Conductance-Based Compartmental Model of the Retina}

Thus far, we have elucidated the time-invariant architecture of our model, the wavelength and spatial-dependence on impinging light. The computational bottleneck of most retinal systems, however, lies in emulation of the high-dimensional spaces that much of the molecular activity within biological cells occurs at. With neural modeling, there are often occasions where, due to an absence of data for a specific cell in a species, one is required to adopt and build neural models whose ionic mechanisms are not from the same class, but from related organisms. The reality is that no complete dataset that wholly characterizes the retinal cellular network of a single animal exists. In light of this, we have procured a substantial collation of experimental data that describes ionic channels, membrane channel densities, and temporal synaptic dynamics for each cell in the retina and performed our own fitting on information that was not previously characterized, in our endeavour to build a unified model with correct biophysical properties for the vertebrate retina. 


\begin{tabular}{|c|c|c|}
\hline \multicolumn{3}{|c|}{ Table 1 - Feature collation for retinal modeling } \\
\hline Species & Function & Literature \\
\hline$\overline{\text { Collated }}$ & Bandpass filtering of rod photoreceptor network & Kamiyama et al. (2009) \\
\hline Collated & Ionic current in rod photoreceptor network & Kamiyama et al. (1996) \\
\hline Salamander & $\begin{array}{l}\text { Voltage- and calcium-activated current of rod cell inner } \\
\text { segment }\end{array}$ & Bader et al. (1982) \\
\hline Lizard & $\begin{array}{l}\text { Calcium and calcium-dependent chloride currents in cone } \\
\text { photoreceptor cells }\end{array}$ & Maricq et al. (1988) \\
\hline Salamander & Ionic channels of cone photoreceptors & Barnes et al. (1989) \\
\hline Toad & Phototransduction in rod cells & Torre et al. (1990) \\
\hline Salamander & The conductance of rod cells & Baylor et al. (1986) \\
\hline Newt & Phototransduction in rod cells & Forti et al. (1989) \\
\hline Turtle & Electrical responses of cone cells & Baylor et al. (1974) \\
\hline Collated & Ionic current in bipolar cells & Usui et al. (1996) \\
\hline Goldfish & Hyperpolarization of activated current in the cell body & Kaneko et al. (1985) \\
\hline Goldfish & Calcium current in the axon terminal & Tachibana et al. (1991) \\
\hline Goldfish & Ca-dependent chloride current in the axon terminal & Tachibana et al. (1993) \\
\hline White bass & Delayed rectifying potassium current in the cell body & Lasater (1988) \\
\hline Axolotl & GABA-induced current in the axon terminal & Attwell et al. (1987) \\
\hline Axolotl & Glutamate-induced current in the dendrite & Attwell et al. (1987) \\
\hline Salamander & Glutamate-induced current in the dendrite & Nawy et al. (1990) \\
\hline Dogfish & Glutamate-induced current in the dendrite & Shiells et al. (1994) \\
\hline Collated & $\begin{array}{c}\text { Ionic channels of AII amacrine cell of the mammalian } \\
\text { retina }\end{array}$ & Smith et al. (1995) \\
\hline Rat & $\begin{array}{l}\text { Action potentials in } \mathrm{Na}^{+} \text {and } \mathrm{K}^{+} \text {channels of AII amacrine } \\
\text { cells }\end{array}$ & Boos et al. (1993) \\
\hline Cat & Transient response in AII amacrine cell to light flashes & Nelson (1982) \\
\hline Rabbit & Transient response in AII amacrine cell to light flashes & Dacheux et al. (1986) \\
\hline Salamander & Impulse encoding mechanisms of ganglion cells & Fohlmeister et al. (1997) \\
\hline Salamander & Repetitive firing of ganglion cells & Fohlmeister et al. (1990) \\
\hline Squid* & $\begin{array}{l}\text { Electric current flow through the surface membrane of a } \\
\text { giant nerve fibre }\end{array}$ & Hodgkin \& Huxley (1952) \\
\hline Collated & $\begin{array}{l}\text { Gap junctions and } i_{h} \text { conductance in the enhancement of } \\
\text { the dynamic range }\end{array}$ & Publio et al. (2009) \\
\hline Salamander & $\begin{array}{l}\text { Reciprocal modulation of calcium dynamics at rod and } \\
\text { cone photoreceptor synapses by nitric oxide }\end{array}$ & Kourennyi et al. (2004) \\
\hline
\end{tabular}

Table 1 depicts the sources of all experimental data that make up the system of nonlinear ODEs from (1) - (3), which is subsequently computed as a system of a total of 272 nonlinear integral equations and parameters in Fig. 1. Some of these sources have utilized their own collations of various species in developing either their models or experimental data, in which case, these references have been grouped in accordance with color. Notably, the squid is the only species on the list which is not classified as posessing a vertebrate retina. However, the HodgkinHuxley conductance-based model, while derived from the squid, can be extended to the vertebrate retina due to the likeness in the ionic mechanisms underlying the initiation and propagation of action potentials [15]. The parameterizable features of electrical and chemical synapses between neurons include the maximum conductance, the reversal potential, the slope in millivolts, and the time constant. The detailed references from Table 1 can be found in the appendix in addition to all equations and variables [12]. 


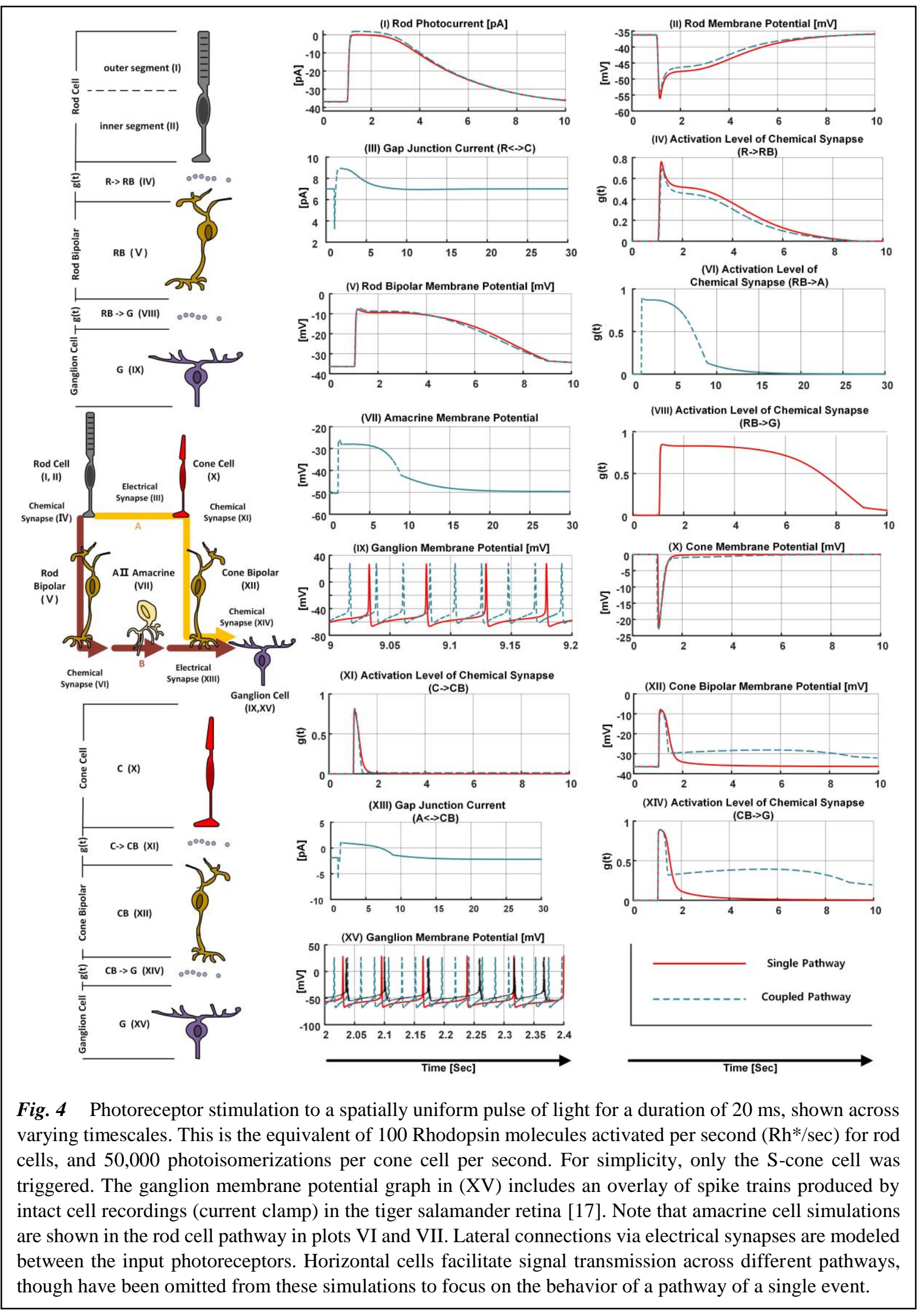




\section{Simulation Platform}

The simulation platform was developed in the $\mathrm{C}$ language, the user interface in Microsoft Foundation Class, and graph plotting using Kst and is recommended for use on Windows 10. Full-scale network simulation is depicted in Fig. 4 with decoupled rod and cone cell pathways, and the coupled pathway which exhibits horizontal lateral connections through electrical synapses, in response to a spatially uniform pulse of light that is activated for a duration of 20 ms. Importantly, the simulation was conducted in real-time at a biological time scale, and all listed measurements in Fig. 4 present a new method to attain benchmarks for vertebrate retina signal extraction under a flexible user-configurable input.

\begin{tabular}{|c|c|c|c|}
\hline \multicolumn{4}{|c|}{ Table 2 - Execution Time as a Function of Time Steps } \\
\hline \multirow{3}{*}{ Time step (s) } & \multicolumn{3}{|c|}{ Normalized Execution Time } \\
\hline & \multicolumn{3}{|c|}{ S-Cone Pathway } \\
\hline & Midpoint & RK4 & ODE15s \\
\hline $1.00 \times 10^{-1}$ & 2.33 & 1.00 & 1.00 \\
\hline $1.00 \times 10^{-2}$ & 1.67 & 2.67 & 168 \\
\hline $1.00 \times 10^{-3}$ & 16.0 & 20.0 & 183.3 \\
\hline $1.00 \times 10^{-4}$ & 180.7 & 231.0 & 375.3 \\
\hline $1.00 \times 10^{-5}$ & 1,182 & 1,738 & 2,139 \\
\hline $1.00 \times 10^{-6}$ & 11,150 & 17,194 & 21,184 \\
\hline \multirow{3}{*}{ Time step (s) } & \multicolumn{3}{|c|}{ Normalized Execution Time } \\
\hline & \multicolumn{3}{|c|}{ Rod Pathway } \\
\hline & Midpoint & RK4 & ODE15s \\
\hline $1.00 \times 10^{-1}$ & 3.25 & 1.00 & 1.00 \\
\hline $1.00 \times 10^{-2}$ & 1.5 & 2.00 & 1,100 \\
\hline $1.00 \times 10^{-3}$ & 13.3 & 16.5 & 1,190 \\
\hline $1.00 \times 10^{-4}$ & 139.8 & 186.3 & 1,268 \\
\hline $1.00 \times 10^{-5}$ & 1,044 & 1,518 & 2,038 \\
\hline $1.00 \times 10^{-6}$ & 10,049 & 14,899 & 15,108 \\
\hline
\end{tabular}

The light input is first passed through a weighted conditional switch in Fig. 1 as determined by the wavelength of light input, and the type of cone cell. The polynomial/linespread response (demonstrated in Fig. 3 under a specific test case) is processed using the integral form of (1) (3), through repeated cycles of iterating time steps until the solution converges. Table 2 provides a comparative analysis of speed between various numerical solvers, namely the classical fourth- 


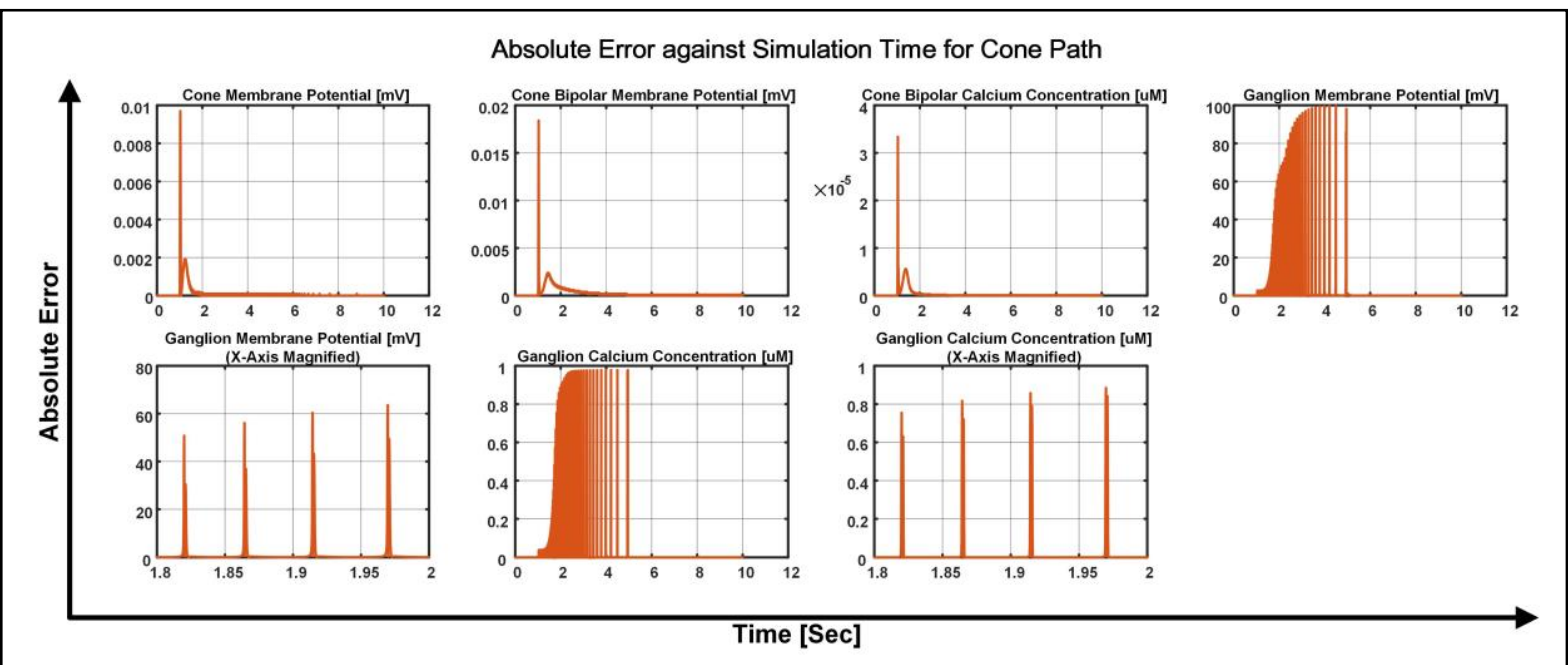

Fig. 5 Absolute error over biological time for the S-cone cell pathway. In all cases, cells display a maximum error at the same time the stimulus is applied for $20 \mathrm{~ms}$.

order Runge-Kutta (RK4) method, the more atypical ODE15s solver, and the midpoint methods - the latter two being our points of comparison for a conventional system of ODEs. In addition to collectively providing the fundamental basis for our retinal model, the studies undertaken in Table 2 are used as a basis of comparison for a qualitative verification of accuracy, while a quantitative analysis is undertaken in Fig. 5. To quantify the accuracy of the proposed model, the benchmark used was the conventional ODE15s solver from the MATLAB ODE suite at a very small time step of $1.00 \times 10^{-9} \mathrm{~s}$. The error for the ganglion membrane potential and the calcium concentration accumulates over time, which arises due to slight misalignments between spike patterns leading to an overstatement of absolute error. When the data is manually timescaled such that peaks of spikes match, the error is bounded and ceases to accumulate.

A range of other conventional ODE solvers were tested (namely, ODE45, ODE23s and ODE113). ODE45 and ODE113 are well-suited for solving non-stiff systems and require unreasonably low time steps for stable and accurate solutions. This significantly increased computation time. ODE15s and ODE 23s are both appropriate for stiff systems, but ODE23s tends to only operate effectively with crude error tolerances. As such, ODE15s was selected as both fastest and most accurate of all the solvers, and we selected only the best performing method as the benchmark for comparison against our proposed system of nonlinear integral equations. Ultimately, the RK4 method is consistently faster than the ODE solver for fixed time steps demonstrating the feasibility of generating real-time predictive retinal measurements.

The values in Table 2 have been normalized to the value of the ODE15s execution time with a time step of $1.00 \times 10^{-1} \mathrm{~s}$. One discrepancy in the results has been highlighted and occurs with the midpoint method which is computationally slower at a time step of $1.00 \times 10^{-1} \mathrm{~s}$ than $1.00 \times 10^{-}$ 
$2 \mathrm{~s}$. This is explained as a result of the time step simply being too large at $1.00 \times 10^{-1} \mathrm{~s}$ to characterize the full spike at the spike-train output of the ganglion cell, which occurs on the order of milliseconds, and can be verified by reference to the ganglion cell simulation shown by curve (XV) in Fig. 4, which depicts both decoupled and coupled retinal pathways of the ganglion membrane potential in millivolts.

\section{Conclusions}

A biologically plausible integral based simulation platform is described in this paper. The simulator is expected to both provide a benchmark for computational neuroscientists who need fast and accurate predictive networks of the retina, and to also assist fundamental studies in mathematics and biomedical sciences that are exploring how the retina is processing information to inspire new ways to introduce sparsity into computer vision. While we are still some distance from a perfect one-to-one model of the retina, we anticipate this simulation platform will help accelerate those studies in order to gain a more in-depth insight into the roles of constituent cells and their associated parameters, and how they may leverage the use of spiking neural networks in assisting other maturing deep learning tasks. Longer-term research motivations can make use of biological real-time simulation for use in bio-hybrid experimentation. The simulator is available for download at the following link: http://ccns.cbnu.ac.kr/paper/1611/retinamodelsimulator.zip.

\section{Acknowledgments}

This work was supported by the National Research Foundation of Korea Grant funded by the Korean Government under Grant NRG-2017R1D1A1A09000613, by the DFAT AustraliaKorea Foundation under Grant AKF00640, and by iDataMap Corporation Pty. Ltd. Australia.

\section{References}

[1] L. McIntosh, N. Maheswaranathan, A. Nayebi, S. Ganguli and S. Baccus, "Deep learning models of the retinal response to natural scenes," in 30th Neural Information Processing Systems (NIPS) Conference, Barcelona, 2016.

[2] D. Saha and A. Mukherjee, "Pervasive computing: A paradigm for the 21st century," Computer, vol. 36, pp. 25-31, March 2003. 
[3] C. Mead, "Neuromorphic electronic systems," Proceedings of the IEEE, vol. 78, no. 10, pp. 1629-1636, March 1990.

[4] P. Lichtsteiner, C. Posch and T. Delbruck, "A 128×128 $120 \mathrm{~dB} 15 \mu$ s Latency Asynchronous Temporal Contrast Vision Sensor," IEEE Journal of Solid-State Circuits, vol. 43, no. 2, pp. 566-576, February 2008.

[5] A. Linares-Barranco, A. Rios-Navarro, R. Tapiador-Morales and T. Delbruck, "Dynamic Vision Sensor integration on FPGA-based CNN accelerators for high-speed visual classification," arXiv:1905.07419 [cs. CV], 2019.

[6] A. Moini, A. Bouzerdoum, K. Eshraghian, A. Yakovleff, X. T. Nguyen, A. Blanksby, R. Beare, D. Abbott and R. E. Bogner, "An Insect Vision-Based Motion Detection Chip," IEEE Journal of Solid-State Circuits, vol. 32, no. 2, pp. 279-284, February 1997.

[7] F. B. Naeini, A. Alali, R. Al-Husari, A. Rigi, M. K. AlSharman, D. Makris and Y. Zweiri, "A Novel Dynamic-Vision-Based Approach for Tactile Sensing Applications," IEEE Transactions on Instrumentation and Measurement (Early Access), May 2019.

[8] J. K. Eshraghian, S. Baek, J.-H. Kim, N. Iannella, K. Cho, Y. S. Goo, H. H. Iu, S.-M. Kang and K. Eshraghian, "Formulation and implementation of nonlinear integral equations to model neural dynamics within the vertebrate retina," International Journal of Neural Systems, vol. 28, no. 7, p. 1850004, September 2018.

[9] V. Kefalov, Y. Fu, N. Marsh-Armstron and K.-W. Yau, "Role of visual pigment properties in rod and cone phototransduction," Nature, vol. 425, no. 6975, pp. 526-531, 2003.

[10] J. K. Eshraghian, K. Cho, C. Zheng, M. Nam, H. H.-C. Iu, W. Lei and K. Eshraghian, "Neuromorphic Vision Hybrid RRAM-CMOS Architecture," IEEE Transactions on Very Large Scale Integration (VLSI) Systems, vol. 26, no. 12, pp. 2816-2829, May 2018.

[11] A. Vanarse, A. Osseiran and A. Rassau, "Neuromorphic Engineering - A Paradigm Shift for Future IM Technologies," IEEE Instrumentation \& Measurement Magazine, vol. 22, no. 2, pp. 4-9, April 2019.

[12] S. Baek and J. K. Eshraghian, "Mathematical Modeling of Retinal Cells," [Online]. Available: http://ccns.cbnu.ac.kr/paper/1605/appendix.pdf.

[13] A. Stockman, D. I. MacLeod and N. E. Johnson, "Spectral sensitivitesof the human cones," JOSA A, vol. 10, no. 12, pp. 2491-2521, December 1993.

[14] B. A. Wandell, Foundations of Vision, Sinauer Associates, 1995.

[15] A. Hodgkin and A. F. Huxley, "A quantitative description of membrane current and its application to conduction and excitation in nerve," The Journal of Physiology, vol. 117, no. 4, pp. 500-544, August 1952.

[16] S. Baek and J. K. Eshraghian, "Retinal Simulator for Neuromorphic Instrumentation," 2019. [Online]. Available: http://ccns.cbnu.ac.kr/paper/1611/retinamodelsimulator.zip .

[17] J. F. Fohlmeister, P. A. Coleman and R. F. Miller, "Modeling the repetitive firing of retinal ganglion cells," Brain research, vol. 510, no. 2, pp. 343-345, 1990. 
Jason K. Eshraghian (S'16-M'19) received the LL.B \& B. Eng, and Ph.D degrees in electrical and electronic engineering from the University of Western Australia, Perth, WA, Australia in 2016 and 2019, respectively. From 2015 to 2016, he was a Research Associate at Chungbuk National University, Cheongju, South Korea working on the Memristive Retina Project, and joined the University of Michigan, Ann Arbor, as a Post-Doctoral Fellow in 2019. His current research interests include memristive systems, inference acceleration and generative adversarial networks. He was awarded the 2019 IEEE Very Large Scale Integration Circuits and Systems Best Paper Award, and the 2019 IEEE Artificial Intelligence Circuits and Systems Conference Best Paper Award, and is a member of the IEEE Neural Systems and Applications Technical Committee.

Seungbum Baek received the B.S. and M.S. degrees in information and communication engineering from Chungbuk National University, Cheongju, South Korea, in 2015 and 2017, respectively, where he is also currently pursuing the Ph.D. degree. His current research interests include mathematical modeling for biomedical engineering applications, VLSI design for security services targeting resource-constrained devices, and embedded systems.

Kyoungrok Cho (S'89-M'92) received the B.S. degree in electronic engineering from Kyoungpook National University, Taegu, Korea, in 1977 and the M.S. and Ph.D. degrees in electrical engineering from University of Tokyo, Tokyo, Japan, in 1989 and 1992, respectively. His current research interests include high speed and low-power circuit designs, SoC platform design for communication systems, and prospective CMOS image sensors, memristor-based circuits, and the design of multilayer systemon-systems technology. He is currently a Professor at the College of Electrical and Computer Engineering, Chungbuk National University, Cheongju, South Korea, where he is also the Director of the IC Design Education Center.

Timothée Levi received the Ph.D degree in Electronics at the University of Bordeaux in 2007. From October 2009 to today, he is an Associate Professor at the University of Bordeaux, France. During this period, from September 2013 to August 2015, he was a Visiting Research Fellow at University of Tokyo, Japan. From April 2017, He is Project Associate Professor at The University of Tokyo. His research interest deals with neuromorphic engineering which aims at designing and using integrated circuits which components and architecture that are neuromimetic for bio-hybrid experiments. 
Takashi Kohno obtained his M.D. in Medicine and Ph.D. in Engineering from University of Tokyo. His research interest is in silicon neurons and neural networks. He is currently Professor at the Institute of Industrial Science, University of Tokyo.

Said Al-Sarawi (S'92-M'96) received the B.Eng. degree (honors) in marine electronics and communication from the Arab Academy for Science, Technology \& Maritime Transport, Alexandria, Egypt, in 1990, and the Ph.D. degree in electrical and electronic engineering from The University of Adelaide, Adelaide, SA, Australia in 2003. He is currently the Director of the Centre for Biomedical Engineering, The University of Adelaide. His current research interests include design techniques for mixed signal systems in microelectronictron and nanoelectronictron and optoelectronic technologies for high-performance radio transceivers, lowpower and low-voltage radio frequency identification systems, data converters, and microelectromechanical systems for biomedical applications. Dr. Al-Sarawi received the Commonwealth Postgraduate Research Award (Industry) and The University of Adelaide Alumni Postgraduate Medal for outstanding academic merit at the postgraduate level.

Philip Leong received the B.Sc., B.E., and Ph.D. degrees from The University of Sydney, Sydney, NSW, Australia, in 1987, 1989, and 1993, respectively. In 1993, he was a Consultant at ST Microelectronics, Milan, Italy. From 1997 to 2009, he was with The Chinese University of Hong Kong, Hong Kong. He is currently a Professor of Computer Systems at the School of Electrical and Information Engineering, The University of Sydney; a Visiting Professor at the Imperial College London, London, U.K.; a Visiting Professor at the Harbin Institute of Technology, Harbin, China; and the Chief Technology Advisor at ClusterTech, Hong Kong

Derek Abbott (M'85-SM'99-F'05) received the B.Sc. (Hons.) degree in physics from Loughborough University, Leicestershire, U.K., in 1982 and the Ph.D. degree in electrical and electronic engineering from The University of Adelaide, Adelaide, SA, Australia. Since 1987, he has been with The University of Adelaide, where he is currently a full Professor with the School of Electrical and Electronic Engineering. His research interests include multidisciplinary physics and electronic engineering applied to complex systems, networks, game theory, energy policy, stochastics, and biophotonics. Prof. Abbott has received a number of awards, including the David Dewhurst Medal (2015), the Barry Inglis Medal (2018), and the M. A. Sargent Medal (2019) for eminence in engineering.

Omid Kavehei is a Senior Lecturer, a SOAR Fellow and a Deputy Director of The University of Sydney Nano Institute. He is with the Faculty of Engineering at The University of Sydney. 
Before this role, he was with Centre for Neural Engineering at The University of Melbourne as a Research Fellow in Microelectronics, where he worked on an Australian engineering flagship project, the Bionic Eye. He received his $\mathrm{PhD}$ in Electronics from the University of Adelaide in 2012 with the Postgraduate University Alumni Medal, a University Doctoral Research Medal and the 2011 South Australian Young Nanotechnology Ambassador award. He was a Visiting Project Scientist at the University of California, Santa Barbara. His research interests include neuromorphic computing for biomedical signal processing and emerging applications of nanoelectronics. 